AIDS, Publish Ahead of Print

DOI: 10.1097/QAD.0000000000002378

\title{
High Soluble CD163 Levels Correlate with Disease Progression and Inflammation in
} Kenyan Children with Perinatal HIV-infection

\section{Short Title}

Soluble CD163 in HIV-infected children

Matthew GENEROSO ${ }^{1}$, Patricia ÁlvareZ ${ }^{1}$, Adam KRAVIETZ1 ${ }^{1}$, Mussa MWAMZUKA ${ }^{2}$, Fatma MARSHED ${ }^{2}$, Aabid AHMED ${ }^{2}$, Alka KHAITAN ${ }^{1,3 *}$

${ }^{1}$ New York University School of Medicine, Department of Pediatrics, Division of Infectious

Diseases, New York, NY, USA; ${ }^{2}$ Bomu Hospital, Mombasa, Kenya, ${ }^{3}$ Indiana University School of Medicine, Ryan White Center for Pediatric Infectious Diseases and Global Health, Indianapolis, IN, USA

* Corresponding author: Alka Khaitan: Indiana University School of Medicine, Ryan White Center for Pediatric Infectious Diseases \& Global Health; 705 Riley Hospital Drive, Suite 3032, Phase 3; Indianapolis, IN 46202; akhaita@iu.edu; phone 317-944-3698

Funding: This study was funded by NIH grant 5K08AI093235-02 to AK. It was also supported by Centers for Disease Control and Prevention (CDC) Co-operative Agreement (5U2GPS002063-03). The contents are solely the responsibility of the authors and do not necessarily represent the official views of the CDC.

This is the author's manuscript of the article published in final edited form as:

Generoso, M., Álvarez, P., Kravietz, A., Mwamzuka, M., Marshed, F., Ahmed, A., \& Khaitan, A. (2019). High soluble CD163 levels correlate with disease progression and inflammation in kenyan children with perinatal hiv-infection. AIDS, Publish Ahead of Print. https://doi.org/10.1097/QAD.0000000000002378 


\section{Abstract}

Objectives: CD163 is a hemoglobin scavenger receptor on monocytes and macrophages, cleaved to soluble CD163 (sCD163) in the plasma following activation. In HIV+ adults, sCD163 is linked to non-AIDS morbidity and predicts mortality, but there is limited data in children. We investigated sCD163 levels in HIV+ children and their correlations with disease progression, immune activation and gut mucosal damage.

Design and Methods: We quantified sCD163 levels in Kenyan children aged 0-20 years with perinatal HIV infection, including 74 ART-naive (ART-) and 64 virally suppressed on ART (ART+), and 79 HIV unexposed-uninfected controls (HIV-). The cohort was divided into age groups 0-5 (younger) and 5-20 (older) years. Correlations between sCD163 and HIV viral load, \%CD8, CD4:CD8 ratio, markers of $\mathrm{T}$ cell activation and proliferation, and gut mucosal damage were also assessed.

Results: ART- children have higher sCD163 levels compared to HIV- and ART+ children ( $\mathrm{p} \leq 0.01)$; $\mathrm{ART}+$ have equivalent sCD163 levels to HIV- children. In a prospective analysis, sCD163 levels decreased in older ART- children after 12 months of treatment $(\mathrm{p}<0.0001)$. Regardless of age, sCD163 levels correlate with clinical disease progression measured by \%CD4 T cells, CD4:CD8 T cell ratios and HIV viral load. Soluble CD163 levels directly correlate with T cell activation markers CD38, HLA-DR, and Ki67 (p $\leq 0.01)$.

Conclusions: High plasma sCD163 levels in HIV+ children correlate with advancing disease and T cell activation. ART initiation normalizes sCD163 levels and may alleviate HIV-related morbidities and improve long-term pediatric outcomes.

Key Words: soluble CD163, HIV, children, immune activation, I-FABP

\section{Introduction}

Copyright @ 2019 Wolters Kluwer Health, Inc. Unauthorized reproduction of this article is prohibited. 
Globally, there are 36.9 million people living with HIV, of which 1.8 million are children ${ }^{[1]}$. With successful antiretroviral drugs, the major cause of HIV-related morbidity and mortality has shifted to non-AIDS complications, including cardiovascular disease and neurocognitive dysfunction $^{[2,3]}$. These co-morbidities are intricately linked to immune activation which begins early in HIV infection due to gut mucosal CD4 T cell destruction allowing microbial products to filter into systemic circulation and trigger immune responses mediated by monocytes and macrophages ${ }^{[4-8]}$. The subsequent chronic inflammation in the innate and adaptive immune systems drives HIV disease progression.

CD163 is a hemoglobin scavenger receptor expressed primarily on monocytes and macrophages ${ }^{[4,9]}$. Upon activation from exposure to pro-inflammatory stimuli such as lipopolysaccharide (LPS), Fc $\gamma$ receptor crosslinking or oxidative stress, membrane-bound CD163 sheds via proteinase-mediated cleavage as soluble CD163 (sCD163) ${ }^{[9]}$. Thus, plasma levels of sCD163 reflect non-specific monocyte and macrophage activation ${ }^{[4,10]}$. Membranebound CD163 endocytoses haptoglobin-hemoglobin complexes and plays a role in erythroblast adhesion and immune sensing of bacteria ${ }^{[4,}{ }^{9]}$. The function of $\mathrm{sCD} 163$ is undefined but has been proposed to be involved in inhibition of lymphocyte proliferation ${ }^{[9,11 \text {, }}$ ${ }^{12]}$. As a hemoglobin scavenger sCD163 also plays an important role in extracellular iron recycling, which inhibits growth of iron-dependent bacterial pathogens ${ }^{[4,29,30]}$.

$\mathrm{HIV}+$ adults have elevated plasma sCD163 levels that persist despite antiretroviral treatment $(\mathrm{ART})^{[4,13-16]}$. High sCD163 plasma concentrations directly correlate with the presence and progression of carotid artery atherosclerosis, noncalcified coronary plaques, and coronary artery stenosis ${ }^{[10,13,16-18]}$. In both human and nonhuman primate studies, monocyte activation is also associated with HIV neuropathogenesis ${ }^{[19,20]}$. Most importantly, sCD163 predicts allcause mortality in adults ${ }^{[10]}$. Thus, in adults, plasma sCD163 levels serve as a biomarker of HIV-related morbidity and mortality.

Copyright $\odot 2019$ Wolters Kluwer Health, Inc. Unauthorized reproduction of this article is prohibited. 
In children, the early onset of inflammation during immunologic and neurologic development leads to additional long-term comorbidities such as delayed cognitive development and early onset cardiovascular disease ${ }^{[21-24]}$, yet there are limited data on plasma sCD163 levels in the pediatric population ${ }^{[25-27]}$. In a cohort of perinatally-infected HIV+ Kenyan children aged 020 years, we report elevated SCD163 plasma concentrations that normalize to levels similar to HIV negative age-matched controls. High sCD163 plasma levels correlate with advancing HIV disease, gut mucosal disruption, and CD4 and CD8 T cell activation, as well as CD4 T cell proliferation.

\section{Materials and Methods}

\section{Participants}

Ethical approval for this study was obtained from New York University and Kenyatta National Hospital/University of Nairobi. Written informed consent and verbal assent was obtained from all participants and/or parents. We enrolled 138 perinatally-infected $\mathrm{HIV}+$ and 79 HIV negative-unexposed children (HIV-) ages 2 months to 20 years from Bomu Hospital in Mombasa, Kenya between 2011-2012. HIV+ children included 74 ART naïve (ART-) and 64 on ART for at least six months (ART+). Individuals with a recent pregnancy or acute illness or active Mycobacterium tuberculosis or malaria infection were ineligible for study entry.

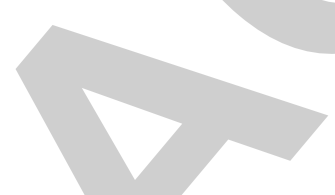

Subjects were subdivided into age groups of 0-5 ("0-5y" or "younger") and 5-20 ("5-20y" or "older") years old based on immunologic maturity. In both age cohorts HIV-, ART-, and ART + were matched for age and gender (Table 1).

\section{Plasma Assays}

Copyright $\odot 2019$ Wolters Kluwer Health, Inc. Unauthorized reproduction of this article is prohibited. 
Plasma levels of sCD163 and Intestinal Fatty Acid Binding Protein (I-FABP) were quantified by ELISA assay using Human sCD163 and I-FABP Duoset kits (R\&D Systems). Plasma samples were diluted 1:100 for sCD163 and 1:1500 for I-FABP assays based on titration assays to fit within the standard curve range. Testing was performed in duplicate with average of duplicate results reported.

\section{Flow Cytometric Studies}

Cryopreserved PBMCs were thawed then evaluated with flow cytometry as described previously ${ }^{[28]}$. For intracellular cytokine staining, cells were activated with phorbol-myristate acetate (PMA) and Ionomicin in the presence of monensin for 5 hours, then fixed, permeabilized (eBioscience) and stained with intracellular antibodies. The following antihuman antibodies were used: CD3, CD4, CD8, CD45RO, CD38, HLA-DR, Ki67 and IL-2. Stained cells were analyzed using LSRII flow cytometer (BD Bioscience) and FlowJo software (Tree Star).

\section{Statistical Analysis}

All statistical analyses were performed using GraphPad Prism software. Comparisons between multiple groups were performed with the Kruskal-Wallis followed by the two-stage linear step-up procedure of Benjamini, Krieger and Yekutieli to correct for multiple comparisons by controlling the False Discovery Rate. Multiple time points were evaluated with Wilcoxon matched-pairs signed-rank test. Correlations were assessed with the Spearman rank test. Threshold of significance for all tests was 0.05 .

\section{Results}

High plasma sCD163 levels in ART- children normalize with treatment 
Plasma sCD163 levels were higher in ART-0-5y than HIV-0-5y $\left(\mathrm{p}=0.004\right.$; Fig A1) and ART ${ }_{0-5 y}$ ( $\mathrm{p}=0.01$; Fig A1). In the older cohort, ART-5-20y also had higher plasma sCD163 levels than HIV-5-20y $\left(\mathrm{p}<0.0001 ;\right.$ Fig A2) and ART ${ }_{5-20 y}(\mathrm{p}<0.0001$; Fig A2). In a subset of ARTchildren who initiated treatment, after $\sim 12$ months of treatment, plasma sCD163 levels remained stable in ART-0-5y children (Fig A3, A5) and decreased in ART-5-20y children ( $<<0.0001$; Fig A4) to levels similar to HIV-5-20y children (Fig A6).

\section{Increased plasma SCD163 levels correlate with HIV disease progression}

To determine the clinical relevance of elevated sCD163 plasma levels, we examined correlations between sCD163 and clinical markers of HIV disease progression. In $\mathrm{HIV}_{0-5 y}$ children, plasma sCD163 levels directly correlated with HIV viral load $(\mathrm{p}=0.004, \mathrm{r}=0.37$; Fig B1) and inversely correlated with $\% \mathrm{CD} 4(\mathrm{p}=0.003, \mathrm{r}=-0.39$; Fig B3) and $\mathrm{CD} 4: \mathrm{CD} 8$ ratios $\left(\mathrm{p}=0.02, \mathrm{r}=-0.32\right.$; Fig B5). In HIV $+_{5-20 y}$ children, plasma $\mathrm{sCD} 163$ levels correlated directly with viral load $(\mathrm{p}<0.0001, \mathrm{r}=0.57$; Fig B2) and inversely with $\% \mathrm{CD} 4(\mathrm{p}=0.004, \mathrm{r}=-0.32$; Fig 1B4) and CD4:CD8 ratios ( $\mathrm{p}=0.007, \mathrm{r}=-0.30$; Fig $\mathrm{B} 6)$.

\section{Plasma sCD163 levels correlate with immune activation and gut mucosal disruption}

Coexpression of CD38 and HLA-DR marks $\mathrm{T}$ cell activation and predicts HIV disease progression. We investigated their correlations with sCD163. In $\mathrm{HIV}+_{0-5 y}$ children, plasma sCD163 levels directly correlate with CD38+HLA-DR+ CD4 T cells $(\mathrm{p}=0.01, \mathrm{r}=0.36$; Fig C1) and CD8 T cells ( $p=0.005, r=0.39$; Fig C3). These correlations were similarly observed in $\mathrm{HIV}_{5-20 y}$ children $(\mathrm{CD} 4: \mathrm{p}<0.0001, \mathrm{r}=0.43$; CD8: $\mathrm{p}=0.008$, $\mathrm{r}=0.29$; Fig C2, C4). We also determined whether SCD163 links to CD4 T cell proliferation by examining associations with Ki67, a marker of activated $\mathrm{T}$ cell proliferation, and IL-2, a cytokine that maintains homeostatic T cell proliferation. Plasma sCD163 concentrations directly correlated with Ki67 levels in memory CD4 $\mathrm{T}$ cells $\left(\mathrm{p}=0.003, \mathrm{r}=0.40\right.$; Fig D1) but not with IL-2 in $\mathrm{HIV}{ }_{0-5 y}$ 
children (Fig D3). In $\mathrm{HIV}_{5-20 y}$ children, sCD163 directly correlated with Ki67 ( $\mathrm{p}=0.0009$, $\mathrm{r}=0.37$; Fig D2) and inversely correlated with IL-2 levels in memory CD4 T cells ( $<<0.0001$, $\mathrm{r}=-0.44$; Fig D4).

We next investigated the relationship between plasma levels of sCD163 and I-FABP, a marker of gut mucosal disruption. In the $\mathrm{HIV}{ }_{0-5 y}$ children, there was no association between these markers (Fig E1), but in $\mathrm{HIV}+_{5-20 y}$ children there was a significant direct correlation $(\mathrm{p}=0.02, \mathrm{r}=0.27$; Fig E2). Last, we investigated whether $\mathrm{sCD} 163$ is associated with another monocyte activation marker, soluble CD14 (sCD14). A direct correlation between the two markers was observed in the HIV $+_{0-5 y}$ children $(\mathrm{p}=0.0006, \mathrm{r}=0.43$; Fig E3), but no correlation was present in the $\mathrm{HIV}+_{5-20 y}$ children (Fig E4).

\section{Discussion}

We demonstrated that plasma sCD163 levels are elevated in untreated HIV+ children beginning in early childhood. In both age cohorts, ART+ children have sCD163 levels similar to HIV- controls. Regardless of age, higher plasma sCD163 levels correlate with worsening disease status, measured by decreasing CD4 $\mathrm{T}$ cell percentage and CD4:CD8 ratio, and increasing HIV viral load. Finally, sCD163 plasma concentrations in HIV+ children correlated with $\mathrm{T}$ cell activation and CD4 $\mathrm{T}$ cell proliferation, suggesting a link between innate and adaptive immunity.

To our knowledge, this is the first report of sCD163 levels in a pediatric cohort aged less than 5 years old with direct comparison to an older cohort. Remarkably, young children exhibited elevation of sCD163 levels similar to older children. Prior studies of sCD163 in HIV+ children include children with median age above 6 years and report high plasma sCD163 levels that decrease with ART. Anaworanich et al. demonstrate in a Thai cohort with median age 6 years, that sCD163 levels in ART+ children were lower than ART- children ${ }^{[27]}$. Tuluc 
et al. report in children aged 9-18 years plasma sCD163 levels significantly decrease after treatment with Raltegravir ${ }^{[26]}$. However, these two studies lack an HIV- control group to evaluate whether sCD163 plasma levels normalize. In our cohort, younger and older children normalized sCD163 levels in both cross-sectional and prospective analyses. Rudy et al. show plasma sCD163 levels significantly decrease after 48 weeks of ART, but remain significantly higher than HIV- $\operatorname{controls}^{[25]}$, as opposed to our observation of similar sCD163 concentrations in ART + and HIV- children. We speculate the discrepancy may reflect different age cohorts, as Rudy's findings in HIV+ youth aged 18-25 years matches previous adult sCD163 studies of persistently high sCD163 levels after treatment. It is possible that after a certain age it is no longer possible to reverse monocyte activation. Thus in children there may be a critical window to initiate ART in order to partially reverse monocyte activation.

In HIV+ adults, sCD163 remains increased despite $\mathrm{ART}^{[4,13-16]}$. These high sCD163 plasma levels have been linked to mortality, demonstrated by Knudsen et al. in a study of 933 virally suppressed HIV-infected adults in Denmark ${ }^{[10]}$. With each milligram per liter increase in sCD163 there was a $6 \%$ increase in mortality ${ }^{[10]}$. Interestingly, plasma sCD163 levels were not predictive of mortality in untreated HIV infection, when active HIV replication drives inflammation. Rather, they were only predictive for virally suppressed subjects whose inflammation is likely due to microbial translocation, coinfections or low-level replication of viral reservoirs ${ }^{[10]}$. While $\mathrm{SCD} 63$ levels were linked to all-cause mortality, the predictive value was slightly higher for cardiovascular causes of death ${ }^{[10,31]}$. Whether sCD163 levels are linked to cardiovascular disease and mortality in children is unknown. We demonstrate that high plasma sCD163 concentrations correlate with worsening disease status, indicated by viremia, $\mathrm{T}$ cell activation, and decreasing $\mathrm{CD} 4$ percentages and $\mathrm{CD} 4: \mathrm{CD} 8$ ratios. In pediatric HIV, clinical parameters are associated with HIV-related morbidity while T cell activation 
has been linked to increased risk of early onset cardiovascular disease and neurocognitive dysfunction. We speculate that monocyte activation may also be correlated with similar morbidity ${ }^{[21-24]}$. In adults, chronic inflammation is triggered by gut mucosal disruption. Interestingly, in our cohort plasma sCD163 levels associated with I-FABP levels in older but not younger children, suggesting there may be cumulative damage to the intestinal mucosa that triggers monocyte activation after longer durations of perinatal HIV infection.

In summary, we demonstrate high plasma sCD163 levels in perinatally-infected HIV+ children that correlate with advancing HIV disease. Antiretroviral therapy reverses elevation of plasma sCD163 levels within 12 months. Plasma sCD163 concentrations correlate with T cell activation and proliferation, linking inflammation in the innate and adaptive immune systems. Early evidence of reversible monocyte activation in children supports the urgent implantation of immediate ART initiation in children as recommended by the WHO to alleviate the global burden of complications secondary to HIV infection and improve overall pediatric health outcomes.

\section{Acknowledgments}

We thank all of the children and families who participated in this study.

\section{Disclosures}

The authors have no financial conflicts of interest.

\section{Author Contributions}

MG performed sCD163 ELISA assays, analyzed data and drafted the manuscript and figures. PA performed I-FABP ELISA assay. AKr performed immune phenotyping studies and analyzed flow cytometry data. MM collected and processed blood samples, performed sCD14 ELISA assays and managed data. FM recruited patients and recorded clinical data. AA 
provided input to study design and oversaw recruitment site. AKh conceptualized and supervised the study, designed experiments, interpreted data and edited the manuscript. 


\section{REFERENCES}

1. UNAIDS. Fact Sheet - World AIDS Day 2017. In; 2017.

2. Wilson EM, Singh A, Hullsiek KH, Gibson D, Henry WK, Lichtenstein K, et al. Monocyte-activation phenotypes are associated with biomarkers of inflammation and coagulation in chronic HIV infection. The Journal of infectious diseases 2014; 210(9):1396-1406.

3. Baker JV, Peng G, Rapkin J, Abrams DI, Silverberg MJ, MacArthur RD, et al. CD4+ count and risk of non-AIDS diseases following initial treatment for HIV infection. AIDS (London, England) 2008; 22(7):841-848.

4. Burdo TH, Lentz MR, Autissier P, Krishnan A, Halpern E, Letendre S, et al. Soluble CD163 made by monocyte/macrophages is a novel marker of HIV activity in early and chronic infection prior to and after anti-retroviral therapy. The Journal of infectious diseases 2011; 204(1):154-163.

5. Brenchley JM, Price DA, Schacker TW, Asher TE, Silvestri G, Rao S, et al. Microbial translocation is a cause of systemic immune activation in chronic HIV infection. Nature medicine 2006; 12(12):1365-1371.

6. Ancuta P, Kamat A, Kunstman KJ, Kim EY, Autissier P, Wurcel A, et al. Microbial translocation is associated with increased monocyte activation and dementia in AIDS patients. PloS one 2008; 3(6):e2516.

7. Freiberg MS, Chang CC, Kuller LH, Skanderson M, Lowy E, Kraemer KL, et al. HIV infection and the risk of acute myocardial infarction. JAMA internal medicine $2013 ; 173(8): 614-622$.

8. Musselwhite LW, Sheikh V, Norton TD, Rupert A, Porter BO, Penzak SR, et al. Markers of endothelial dysfunction, coagulation and tissue fibrosis 
independently predict venous thromboembolism in HIV. AIDS (London, England) $2011 ; 25(6): 787-795$.

9. Moller HJ. Soluble CD163. Scandinavian journal of clinical and laboratory investigation 2012; 72(1):1-13.

10. Knudsen TB, Ertner G, Petersen J, Moller HJ, Moestrup SK, Eugen-Olsen J, et al. Plasma Soluble CD163 Level Independently Predicts All-Cause Mortality in HIV-1-Infected Individuals. The Journal of infectious diseases 2016; 214(8):11981204.

11. Frings W, Dreier J, Sorg C. Only the soluble form of the scavenger receptor CD163 acts inhibitory on phorbol ester-activated T-lymphocytes, whereas membrane-bound protein has no effect. FEBS letters 2002; 526(1-3):93-96.

12. Hogger P, Sorg C. Soluble CD163 inhibits phorbol ester-induced lymphocyte proliferation. Biochemical and biophysical research communications 2001; 288(4):841-843.

13. Burdo TH, Lo J, Abbara S, Wei J, DeLelys ME, Preffer F, et al. Soluble CD163, a novel marker of activated macrophages, is elevated and associated with noncalcified coronary plaque in HIV-infected patients. The Journal of infectious diseases 2011; 204(8):1227-1236.

14. O'Halloran JA, Dunne E, Gurwith M, Lambert JS, Sheehan GJ, Feeney ER, et al. The effect of initiation of antiretroviral therapy on monocyte, endothelial and platelet function in HIV-1 infection. HIV medicine 2015; 16(10):608-619.

15. Beltran LM, Munoz Hernandez R, de Pablo Bernal RS, Garcia Morillo JS, Egido J, Noval ML, et al. Reduced sTWEAK and increased sCD163 levels in HIV-infected patients: modulation by antiretroviral treatment, HIV replication and HCV coinfection. PloS one 2014; 9(3):e90541.

Copyright @ 2019 Wolters Kluwer Health, Inc. Unauthorized reproduction of this article is prohibited. 
16. McKibben RA, Margolick JB, Grinspoon S, Li X, Palella FJ, Jr., Kingsley LA, et al. Elevated levels of monocyte activation markers are associated with subclinical atherosclerosis in men with and those without HIV infection. The Journal of infectious diseases 2015; 211(8):1219-1228.

17. Fitch KV, Srinivasa S, Abbara S, Burdo TH, Williams KC, Eneh P, et al. Noncalcified coronary atherosclerotic plaque and immune activation in HIVinfected women. The Journal of infectious diseases 2013; 208(11):1737-1746.

18. Hanna DB, Lin J, Post WS, Hodis HN, Xue X, Anastos K, et al. Association of Macrophage Inflammation Biomarkers With Progression of Subclinical Carotid Artery Atherosclerosis in HIV-Infected Women and Men. The Journal of infectious diseases 2017; 215(9):1352-1361.

19. Burdo TH, Soulas C, Orzechowski K, Button J, Krishnan A, Sugimoto C, et al. Increased monocyte turnover from bone marrow correlates with severity of SIV encephalitis and CD163 levels in plasma. PLoS Pathog 2010; 6(4):e1000842.

20. Fischer-Smith T, Bell C, Croul S, Lewis M, Rappaport J. Monocyte/macrophage trafficking in acquired immunodeficiency syndrome encephalitis: lessons from human and nonhuman primate studies. $J$ Neurovirol 2008; 14(4):318-326.

21. Alvarez P, Mwamzuka M, Marshed F, Kravietz A, Ilmet T, Ahmed A, et al. Immune activation despite preserved CD4 $\mathrm{T}$ cells in perinatally HIV-infected children and adolescents. PloS one 2017; 12(12):e0190332.

22. Newell ML, Coovadia H, Cortina-Borja M, Rollins N, Gaillard P, Dabis F, et al. Mortality of infected and uninfected infants born to HIV-infected mothers in Africa: a pooled analysis. Lancet 2004; 364(9441):1236-1243.

23. Pilakka-Kanthikeel S, Huang S, Fenton T, Borkowsky W, Cunningham CK, Pahwa S. Increased gut microbial translocation in HIV-infected children persists in 
virologic responders and virologic failures after antiretroviral therapy. The Pediatric infectious disease journal 2012; 31(6):583-591.

24. Willen EJ, Cuadra A, Arheart KL, Post MJ, Govind V. Young adults perinatally infected with HIV perform more poorly on measures of executive functioning and motor speed than ethnically matched healthy controls. AIDS care 2017; 29(3):387-393.

25. Rudy BJ, Kapogiannis BG, Worrell C, Squires K, Bethel J, Li S, et al. Immune Reconstitution but Persistent Activation After 48 Weeks of Antiretroviral Therapy in Youth With Pre-Therapy CD4 >350 in ATN 061. Journal of acquired immune deficiency syndromes (1999) 2015; 69(1):52-60.

26. Tuluc F, Spitsin S, Tustin NB, Murray JB, Tustin R, 3rd, Schankel LA, et al. Decreased PD-1 Expression on CD8 Lymphocyte Subsets and Increase in CD8 Tscm Cells in Children with HIV Receiving Raltegravir. AIDS research and human retroviruses 2017; 33(2):133-142.

27. Ananworanich J, Kerr SJ, Jaimulwong T, Vibol U, Hansudewechakul R, Kosalaraksa $\mathrm{P}$, et al. Soluble CD163 and monocyte populations in response to antiretroviral therapy and in relationship with neuropsychological testing among HIV-infected children. Journal of virus eradication 2015; 1(3):196-202.

28. Foldi J, Kozhaya L, McCarty B, Mwamzuka M, Marshed F, Ilmet T, et al. HIVInfected Children Have Elevated Levels of PD-1+ Memory CD4 T Cells With Low Proliferative Capacity and High Inflammatory Cytokine Effector Functions. The Journal of infectious diseases 2017; 216(6):641-650.

29. Madsen M, Moller HJ, Nielsen MJ, Jacobsen C, Graversen JH, van den Berg T, et al. Molecular characterization of the haptoglobin.hemoglobin receptor CD163. 
Ligand binding properties of the scavenger receptor cysteine-rich domain region. The Journal of biological chemistry 2004; 279(49):51561-51567.

30. Weaver LK, Hintz-Goldstein KA, Pioli PA, Wardwell K, Qureshi N, Vogel SN, et al. Pivotal advance: activation of cell surface Toll-like receptors causes shedding of the hemoglobin scavenger receptor CD163. Journal of leukocyte biology 2006; 80(1):26-35.

31. Hunt PW. Soluble CD163 and Clinical Outcomes in Treated HIV Infection: Insights Into Mechanisms. The Journal of infectious diseases 2016; 214(8):11321133. 


\section{FIGURE LEGENDS}

\section{Figure 1: Soluble CD163 levels in HIV-infected children}

Children aged 0-5 years are shown in red and 5-20 years in blue. (A) Left (A1-2): comparison of plasma sCD163 concentrations in HIV unexposed-uninfected (HIV-), HIV-infected untreated (ART-), and HIV-infected treated (ART+) children. Center (A3-4): plasma sCD163 levels in ART- children who began treatment before (T0) and 12 months after (T1) treatment initiation. Right (A5-6): comparison between sCD163 levels in ART- at T0 and T1 and HIV- children. (B) Correlation between plasma sCD163 concentrations and HIV viral load (B1-2), percent CD4 T cells (B3-4), and CD4:CD8 ratio (B5-6) in HIV+ children. Correlation between plasma sCD163 concentrations and (C) percentage of CD38+HLA-DR+ cells on CD4 (C1-2) and CD8 (C3-4) T cells, (D) Ki67 (D1-2) and IL-2 (D3-4) levels in CD45RO+ CD4 T cells, and (E) plasma intestinal fatty acid binding protein (E1-2) and SCD14 (E3-4) levels in HIV+ children.
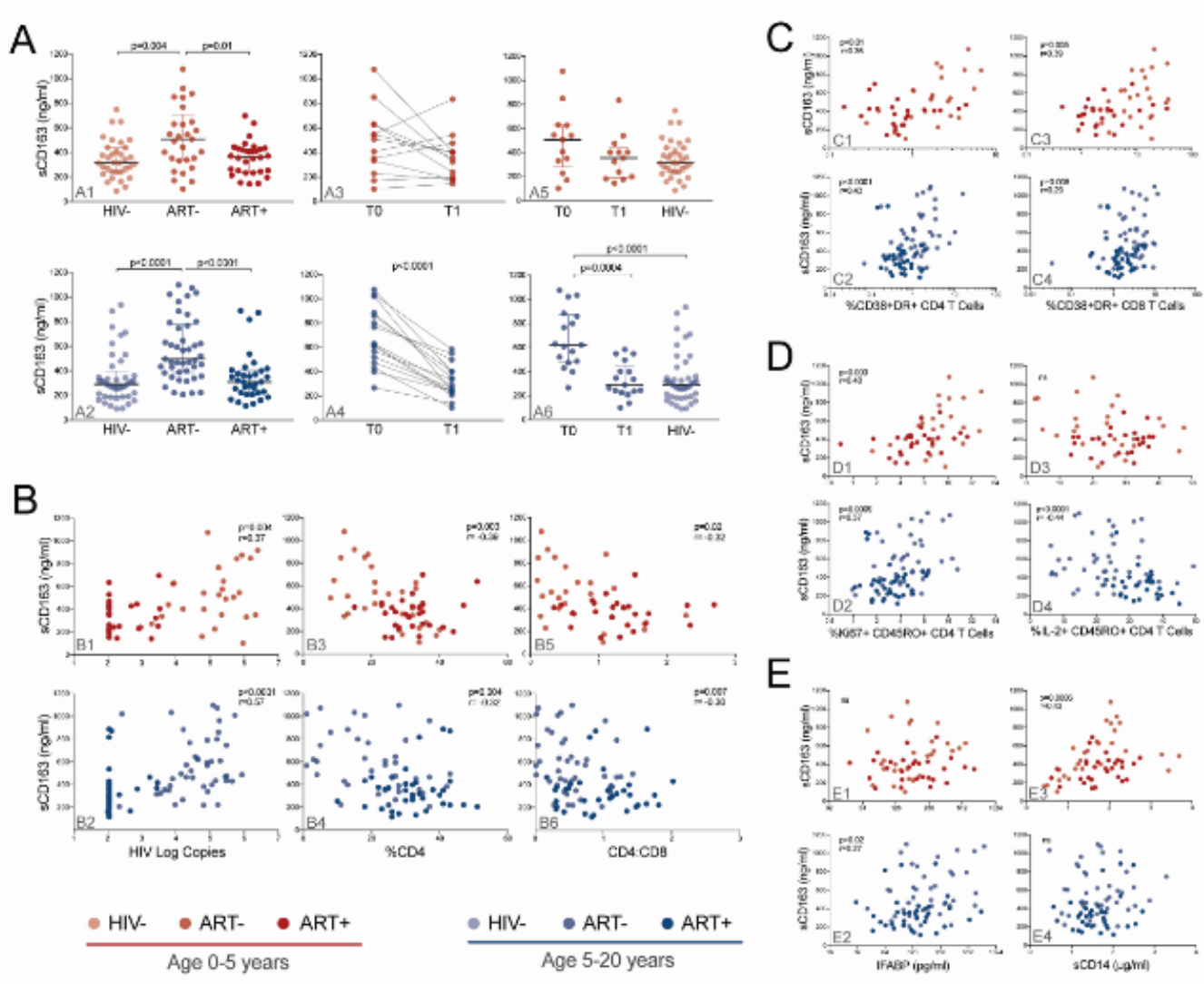

E
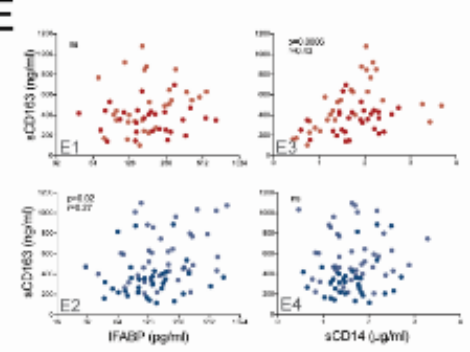
Table 1. Subject Characteristics

\begin{tabular}{|c|c|c|c|c|}
\hline & HIV- & ART- & ART+ & $\mathbf{p}$ \\
\hline \multicolumn{5}{|c|}{ YOUNGER COHORT (0-5 years) } \\
\hline $\mathbf{n}$ & 34 & 29 & 30 & \\
\hline Age (years)* & $2.5(1.2-3.5)$ & $2.0(1.1-3.2)$ & $3.1(2.2-3.5)$ & $\mathrm{NS}^{\mathrm{a}}$ \\
\hline Female (n) & $20(55 \%)$ & $17(56 \%)$ & $12(40 \%)$ & $\mathrm{NS}^{\mathrm{b}}$ \\
\hline$\%$ CD4* & $33(29-38)$ & $21(14-32)$ & $32(27-35)$ & $\mathrm{p}=0.0001^{\mathrm{a}}$ \\
\hline HIV log copies/ml* & & $5.3(4.1-5.8)$ & $2.0(2.0-2.7)$ & $\mathrm{p}<0.0001^{\mathrm{c}}$ \\
\hline \multicolumn{5}{|c|}{ OLDER COHORT (5-20 years) } \\
\hline $\mathbf{n}$ & 45 & & 34 & \\
\hline Age (years)* & $12(9.4-16)$ & $11(8.6-14)$ & $12(8.3-13)$ & $\mathrm{NS}^{\mathrm{a}}$ \\
\hline Female (n) & $18(40 \%)$ & $25(57 \%)$ & $20(60 \%)$ & $\mathrm{NS}^{\mathrm{b}}$ \\
\hline$\%$ CD4* & $38(33-42)$ & $24(13-28)$ & $32(26-40)$ & $\mathrm{p}<0.0001^{\mathrm{a}}$ \\
\hline HIV log copies/ml* & & $4.8(4.2-5.2)$ & $2.0(2.0-2.0)$ & $\mathrm{p}<0.0001^{\mathrm{c}}$ \\
\hline
\end{tabular}

*Median values with upper and lower quartile range

(a) Kruskal-Wallis test; (b) Chi-Square test; (c) two-sided Mann-Whitney test 